\title{
Long-Term In Vivo Imaging of Dendritic Spines in the Hippocampus Reveals Structural Plasticity
}

\author{
Ligang Gu, Stefanie Kleiber, Lena Schmid, Felix Nebeling, Miriam Chamoun, Julia Steffen, Jens Wagner, \\ and Martin Fuhrmann \\ German Center for Neurodegenerative Diseases (DZNE), Bonn, Germany
}

\begin{abstract}
Hippocampal function is important for learning and memory. During memory processing, hippocampal CA1 neurons play a crucial role by integrating excitatory synaptic input from CA3 and the entorhinal cortex. These neurons receive excitatory input almost exclusively on dendritic spines. The formation and elimination — structural plasticity — of dendritic spines reflect wiring changes within the hippocampal network. Despite the relevance of the hippocampus in learning and memory, most in vivo data on structural plasticity derive from cortical regions. We established a chronic hippocampal window approach using two-photon microscopy to visualize dendritic spines throughout all CA1 hippocampal layers and over a time course of weeks. Moreover, even granule cells in dentate gyrus could be reliably detected. We found that the spine density in stratum radiatum $(\sim 1.1$ per micrometer) remained stable over weeks. However, a small fraction (3.4\%) of spines were formed and eliminated between imaging sessions, which demonstrated that spines of CA1 neurons exhibit structural plasticity in adult mice. In addition, we tested for possible inflammatory or behavioral side effects of hippocampal window implantation. Mice exhibited a transient increase in microgliosis and astrogliosis, which declined within a few weeks. We did not detect any difference in behavioral performance in an open-field and contextual fear-conditioning paradigm. In conclusion, hippocampal long-term two-photon imaging revealed structural plasticity of dendritic spines in CA1 pyramidal neurons. This approach may provide a powerful tool to analyze changes in neuronal network rewiring during hippocampal learning and memory processes in health and disease.
\end{abstract}

Key words: behavior; dendritic spines; hippocampus; in vivo imaging; structural plasticity; two-photon imaging

\section{Introduction}

Dendritic spines harbor the postsynaptic compartments of excitatory synapses. The formation and elimination of dendritic spines are the basis for structural remodeling of neuronal networks and take place in response to experience and learning in the cortex (Hübener and Bonhoeffer, 2010). In vitro, changing synaptic transmission by the induction of long-term potentiation induces the formation of new spines (Engert and Bonhoeffer, 1999), whereas long-term depression results in elimination of dendritic spines (Nägerl et al., 2004; Zhou et al., 2004; Wiegert and Oertner, 2013). Two-photon in vivo imaging in transgenic mice with a sparse neuronal expression of yellow fluorescent protein (YFP; Feng et al., 2000) made it possible to study structural

\footnotetext{
Received April 10, 2014; revised Aug. 7, 2014; accepted Sept. 3, 2014

Author contributions: J.W. and M.F. designed research; L.G., S.K., L.S., M.C., and J.S. performed research; L.G., S.K., L.S., F.N., and J.S. analyzed data; M.F. wrote the paper.

This work was supported by the Deutsche Forschungsgemeinschaft (KF0 177, SFB1089) and by the German Center for Neurodegenerative Diseases, Canadian Institutes of Health Research, and the Health Research Board/ Science Foundation Ireland through the Network of Centres of Excellence in Neurodegeneration initiative (www. coen.org). We thank Stefan Remy and Walker Jackson, for helpful discussion on the manuscript, and the light microscopy facility of the German Center for Neurodegenerative Diseases for constant support.

The authors declare no competing financial interests.

Correspondence should be addressed to Martin Fuhrmann, German Center for Neurodegenerative Diseases (DZNE), Bonn, Germany, c/o Biomedical Center, Sigmund-Freud-Str. 25, 53127 Bonn, Germany. E-mail: martin.fuhrmann@dzne.de.

DOI:10.1523/JNEUROSCI.1464-14.2014

Copyright $\odot 2014$ the authors $\quad 0270-6474 / 14 / 3313948-06 \$ 15.00 / 0$
}

plasticity of spines in the somatosensory cortex (Grutzendler et al., 2002; Trachtenberg et al., 2002; Holtmaat et al., 2005). Since then, structural plasticity of dendritic spines has been analyzed in different mouse models and in response to different stimuli. Monocular deprivation leads to profound changes in spine formation and elimination in the visual cortex, while spine densities remain unchanged (Keck et al., 2008). Upon motor learning and fear conditioning, structural changes of spines can be detected in the motor cortex and prefrontal cortex (Yang et al., 2009; Lai et al., 2012) and represent learning-induced structural plasticity in the cortex. In mouse models of neurodegenerative diseases, such as Alzheimer's disease or prion disease, spine loss is a prominent feature of neuronal network changes that parallel cognitive decline (Tsai et al., 2004; Spires et al., 2005; Fuhrmann et al., 2007; Bittner et al., 2010).

While all these studies investigated the superficial cortical layers that primarily process sensory information, deeper brain areas, such as the hippocampus, have not been analyzed for structural changes of dendritic spines over periods of days to weeks. As depth penetration of two-photon in vivo imaging is limited to $700 \mu \mathrm{m}$, this represents a plausible explanation of why only superficial cortical layers have been analyzed. To circumvent this, Mizrahi and colleagues developed an acute hippocampal window preparation, where the cortex above the hippocampus was removed, to investigate dendritic spines in vivo over a period of hours (Mizrahi et al., 2004). Later, the manufacturing of 
gradient-index lenses suitable for two-photon imaging provided access to the hippocampus (Barretto et al., 2009). Recently, $\mathrm{Ca}^{2+}$-imaging with a genetically encoded calcium sensor was used to record place cell activity in the hippocampus (Dombeck et al., 2010). However, high-resolution long-term in vivo imaging of dendritic spines in the hippocampus has not been performed until now. To understand the structural remodeling and rewiring of the hippocampal neuronal network, we analyzed the formation and elimination of dendritic spines of CA1 neurons over weeks in the stratum radiatum in vivo.

\section{Materials and Methods}

Mice. Adult transgenic YFP (YFP-H) mice at the age of 6-7 months (Feng et al., 2000; The Jackson Laboratory) were used for the in vivo imaging experiments. Mice with the same C57BL/6 background were used for the behavior experiments, gliosis quantification, and immediate early gene (iEG) expression analysis. The mice were group housed, separated by gender under specified pathogen-free conditions, and similar numbers of both genders were used. The light/dark cycle was 12/12 h and the humidity and temperature were kept constant (40\% relative humidity; $22^{\circ} \mathrm{C}$ ). All procedures were in accordance with the institutional guidelines of the German Center for Neurodegenerative Diseases and an animal experimental protocol approved by the government of North Rhine Westphalia.

Hippocampal window surgery. Mice were anesthetized with an intraperitoneal injection of ketamine/xylazine $(0.13 / 0.01 \mathrm{mg} / \mathrm{g}$ bodyweight). Additionally, mice received a subcutaneous dose of buprenorphine $(0.05$ $\mathrm{mg} / \mathrm{kg})$ for analgesia and dexamethason $(0.2 \mathrm{mg} / \mathrm{kg})$ to reduce swelling and inflammation. The eyes were covered with eye ointment (Bepanthen) to prevent drying. Mice were placed on a heating blanket to maintain the body temperature. The depth of anesthesia and analgesia was tested with the toe-pinch withdrawal reflex. Subsequently, mice were fixed to a stereotactic frame and the skin of the head was disinfected with $70 \%$ ethanol. The skin above the right part of the skull bone was removed with surgical scissors. After drying of the skull bone with sterile cotton wool wads, a 3-mm-diameter circular piece of the right skull bone was removed (anteroposterior, $-2.2 \mathrm{~mm}$; mediolateral, $+1.8 \mathrm{~mm}$ relative to bregma) using a dental drill. This position was chosen to access the dorsal hippocampus. The dura was carefully removed and the cortex above the hippocampus was aspirated with a 27 gauge needle attached to a $20 \mathrm{ml}$ syringe with flexible tubing. When the external capsule of the hippocampus was reached, the aspiration was stopped and the remaining part of the cortex was carefully peeled away to leave the external capsule intact. Subsequently, the craniotomy was rinsed with sterile PBS and a sterile custom-made metal tube $(\varnothing, 3 \mathrm{~mm}$; height, $1.5 \mathrm{~mm})$ sealed with a circular coverslip [ $\varnothing, 3 \mathrm{~mm}$; height, $0.17 \mathrm{~mm}(\# 1)]$ was inserted and glued to the skull bone with dental acrylic. The coverslip was glued on the metal tube with UV-curable adhesive (Norland Products). A small metal plate with a thread was glued on the skull next to the tube to ensure repetitive repositioning of the mouse under the microscope (Fuhrmann et al., 2007; Dombeck et al., 2010). The process of cortex aspiration and window surgery remained basically the same as described by Dombeck et al. (2010). The main difference was to omit silicone (Kwik-Sil) connecting the hippocampal surface and the cover glass at the bottom of the implanted metal tube. Kwik-Sil was meant to reduce movement artifacts with the secondary effect of decreasing the resolution. After surgery, mice received analgesic doses of buprenorphine $(0.05 \mathrm{mg} / \mathrm{kg}$, s.c.) for the following $3 \mathrm{~d}$ and were allowed to recover from surgery for 4 weeks before imaging started.

Imaging. Fluorescent images of fixed brain slices were acquired on a confocal laser scanning microscope (LSM 700, Zeiss) equipped with an immersion objective $40 \times 1.3$ numerical aperture Plan-Apochromat. YFP was excited at $488 \mathrm{~nm}$ and detected at $500-550 \mathrm{~nm}$. To determine dendritic spine density of YFP-positive CA1 neurons, we acquired confocal image stacks spanning $10 \mu \mathrm{m}$ in $z$-dimension ( $z$-spacing, $0.5 \mu \mathrm{m}$ ) and covering the entire dendritic element (pixel size, $0.1 \mu \mathrm{m} /$ pixel). Images for Iba-1, GFAP, and c-Fos were acquired with the same microscope and a $20 \times 0.8$ numerical aperture air objective (pixel size, $0.4 \mu \mathrm{m} / \mathrm{pixel}$ ).
Alexa 488 or 647 were excited with a 488 or $638 \mathrm{~nm}$ laser and detected with filters (band-pass 500-550 $\mathrm{nm}$ or low-pass $560 \mathrm{~nm}$ ). DAPI was excited at $405 \mathrm{~nm}$ and emission was detected at $460-480 \mathrm{~nm}$.

For in vivo imaging, mice were anesthetized with an intraperitoneal injection of ketamine/xylazine $(0.13 / 0.01 \mathrm{mg} / \mathrm{g}$ bodyweight). The eyes were protected with eye ointment (Bepanthen) and the mouse was placed in a custom-made stereotactic frame using the implanted metal plate as an anchor point. A heating pad $\left(37^{\circ} \mathrm{C}\right)$ was placed under the mouse to maintain the body temperature. Subsequently, the stereotactic frame was placed on a motorized XY-table of an upright microscope (TrimScope II, LaVision Biotech) equipped with a two-photon laser (Cameleon Ultra II, Coherent). A $16 \times 0.8$ numerical aperture water-immersion objective with a working distance of $3 \mathrm{~mm}$ (Nikon) was used for imaging. The two-photon laser was tuned to a wavelength of $920 \mathrm{~nm}$ with a maximum output power of $50 \mathrm{~mW}$ to prevent photo damage. YFP was excited at 920 $\mathrm{nm}$ and the emitted light was detected with a gallium arsenide phosphide detector. In the first imaging session, a picture of the vasculature was taken. The unique pattern of the vasculature enabled subsequent repositioning of the mouse under the microscope. The fine positioning was performed with the motorized XY-table of the microscope by aligning the two-photon image to the first time-point image of neurons, dendrites, and spines. A $z$-stack $(x, y, z: 100 \times 100 \times 60 \mu \mathrm{m})$ with a pixel size of $0.09 \mu \mathrm{m} /$ pixel and a $z$-spacing of $1 \mu \mathrm{m}$ was acquired at each time point starting $50 \mu \mathrm{m}$ below the pyramidal cell layer. An overview stack $(x, y, z$ : $400 \times 400 \times 300 \mu \mathrm{m} ; 5 \mu \mathrm{m} z$-step; pixel size, $0.4 \mu \mathrm{m} /$ pixel) was acquired at the end of the first and last imaging sessions. The imaging sessions lasted for $\leq 60 \mathrm{~min}$ and mice were placed back to their home cages where they woke up.

Behavior. The open-field test was conducted in an opened plastic box $(25 \times 25 \times 30 \mathrm{~cm})$ composed of white walls and a white, roughened floor. Mice were placed in the center of the chamber and allowed to explore the environment for $10 \mathrm{~min}$. The traveled distance was analyzed automatically with EthoVision XT (Noldus). For the fear-conditioning experiment, the training and retrieval were conducted in a chamber $(21.5 \times 20 \times 25 \mathrm{~cm})$ composed of transparent plastic walls and a stainless-steel grid floor connected to an aversive stimulator/scrambler (Med Associates). For the conditioning, mice were placed in the chamber for $120 \mathrm{~s}$ before the first foot shock was delivered $(0.75 \mathrm{~mA}, 2 \mathrm{~s})$. With an intershock period of $60 \mathrm{~s}$, two more shocks were applied and mice were returned to their home cage $60 \mathrm{~s}$ after the third shock. To analyze $c$-fos expression, mice were killed 90 min later. For memory retrieval, mice were placed in the conditioning chamber $48 \mathrm{~h}$ after the fear conditioning and allowed to explore the context for $5 \mathrm{~min}$. During training and retrieval, mice were video recorded. The distance traveled was analyzed automatically with EthoVision XT (Noldus). In addition, an experimenter blind to the experimental groups manually determined the cumulative duration of freezing behavior. Freezing was defined as the complete absence of movement, except for breathing.

Histology and immunohistochemistry. Mice were transcardially perfused with PBS, pH 7.4, followed by $4 \%$ paraformaldehyde (PFA) for 5 min. Brains were removed and fixed overnight in $4 \%$ PFA. Subsequently, immunohistochemical staining was performed as described by Gogolla et al. (2006) with some adaptations. Hundred-micrometer-thick sections were cut on a vibratome (Leica VT 1200, Leica). These sections were permeabilized in $2 \%$ Triton X-100 overnight. Subsequently, free-floating sections were stained with an antibody raised against Iba-1 (rabbit serum, $1 \mu \mathrm{g} / \mathrm{ml}$, Wako), GFAP (rabbit serum, 1:500; Life Technologies), or c-Fos (rabbit serum, 1:500; Santa Cruz Biotechnology). Secondary labeling was performed with an anti-rabbit secondary antibody Alexa 488 (Iba-1, c-Fos) or Alexa 647 (GFAP). A counterstaining was performed using the nuclear marker DAPI (1:1000; Life Technologies) or the neuron-specific marker NeuN (1:1000; Millipore).

Image analysis. The number of Iba1-positive and GFAP-positive cells was counted in the contralateral and ipsilateral hemispheres of the implanted hippocampus window. Three $100-\mu \mathrm{m}$-thick coronal slices at the level of the hippocampus window were analyzed. The density of Iba1positive and GFAP-positive cells was measured in a rectangular area $(600 \times 240 \mu \mathrm{m})$ including stratum oriens, pyramidale, and radiatum of both hippocampi. 
a

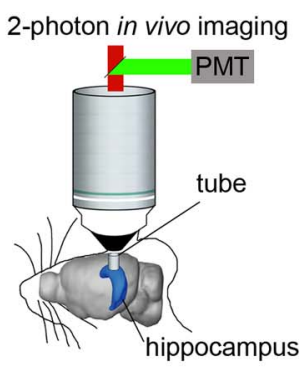

b

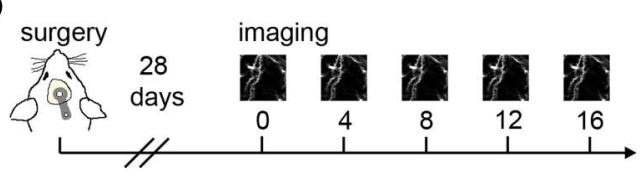

C
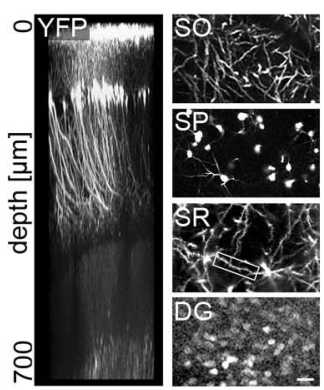

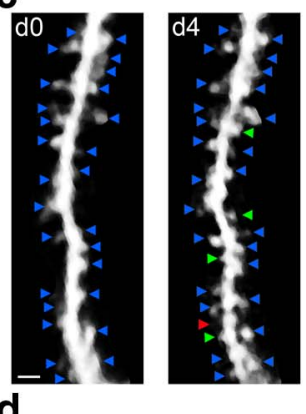

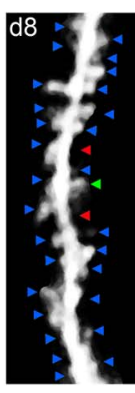
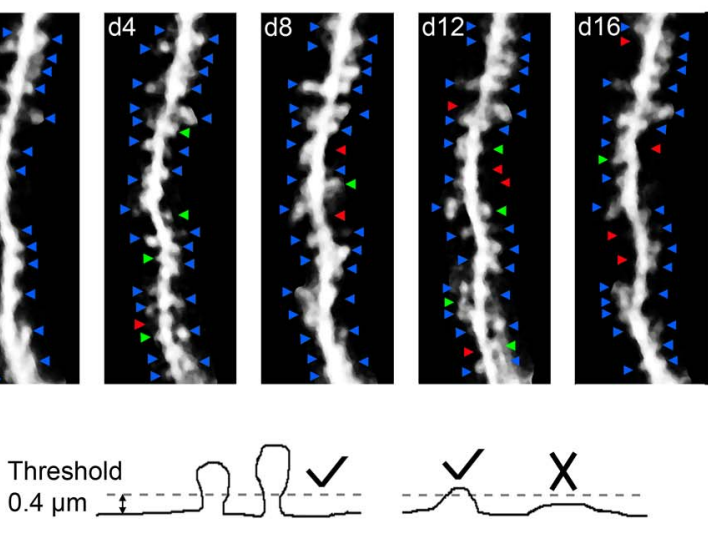

e

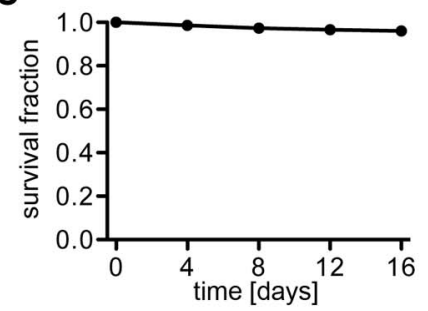

f

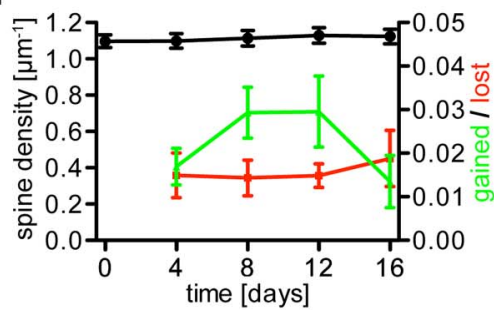

g

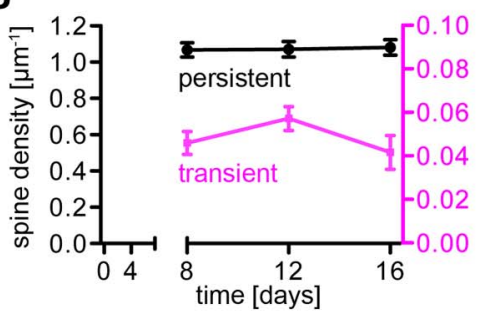

Figure 1. Structural plasticity of dendritic spines in the hippocampus. $\boldsymbol{a}$, Left, Schematic illustrating two-photon in vivo imaging in the hippocampus. Right, Side projection of a $z$-stack acquired up to a depth of $700 \mu \mathrm{m}$ below the hippocampal surface and maximum intensity projections from different depths in stratum oriens (S0), stratum pyramidale (SP), stratum radiatum (SR), and dentate gyrus (DG). $\boldsymbol{b}$, Experimental timeline from surgery until imaging. $\boldsymbol{c}$, Time-lapse images of a radial oblique dendrite over a period of $16 \mathrm{~d}$. Note labeled stable spines (blue arrowheads), gained spines (green arrowheads), and lost spines (red arrowheads). $\boldsymbol{d}$, Schematic with two examples for spine scoring procedure. $\boldsymbol{e}$, Sixteen-day survival fraction of spines. $\boldsymbol{f}$, Spine density of radial oblique dendrites in vivo (black) and densities of lost (red) and gained (green) spines. $\boldsymbol{g}$, Density of transient and persistent spines. $\boldsymbol{e}-\boldsymbol{g}: n=3660$ spines in 5 mice. Scale bars: $\boldsymbol{a}, 20 \mu \mathrm{m} ; \boldsymbol{c}, 2 \mu \mathrm{m}$.

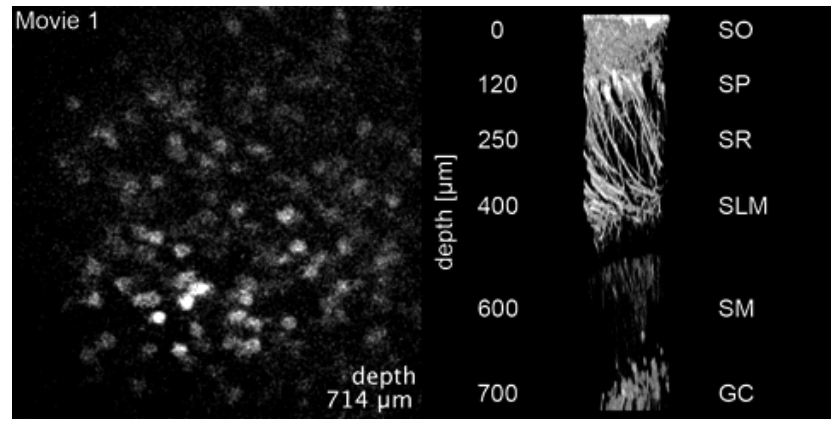

Movie 1. $\quad z$-scan through the hippocampus and 3D-rendered model. Left, Two-photon in vivo imaging through the hippocampus starting at the top of the stratum oriens $(0 \mu \mathrm{m})$ down to the granule cell layer of the dentate gyrus $(700 \mu \mathrm{m})$. Right, $3 \mathrm{D}$ reconstruction of the $z$-scan. SO, Stratum oriens; SP, pyramidale; SR, radiatum; SLM, lacunosum moleculare;SM, moleculare; $\mathrm{GC}$, granule cell layer.

The number of c-Fos-positive and NeuN-positive CA1 neurons was analyzed in both hemispheres of three $100-\mu \mathrm{m}$-thick coronal sections. Cells were counted within a rectangle $(100 \times 200 \mu \mathrm{m})$ that was placed below the hippocampal window and in the corresponding contralateral hemisphere. All images were background corrected by subtracting the background plus twice the SD of pixel intensities within the background region of interest.

To determine dendritic spine density of YFP-positive CA1 neurons in the hippocampus of fixed brain slices, three subsequent rostral-directed $100-\mu \mathrm{m}$-thick slices starting from $-2 \mathrm{~mm}$ from bregma for each mouse were analyzed. In each slice, spine number was counted on 8-10 radial oblique dendrites. The images were median filtered and maximum intensity projected. All laterally emanating dendritic spines were counted and the length of the dendrite was measured. The spine density was calculated as the number of spines divided by the dendritic length in micrometers. These procedures were performed using ZEN (Zeiss, software version 2010).

The spine density in vivo was determined similarly as described before (Holtmaat et al., 2005, 2009; Fuhrmann et al., 2007). In each animal, 5-10 dendrites of $20-50 \mu \mathrm{m}$ length were analyzed. Spines that laterally emanated from the dendrite were counted by manually scrolling through the $z$-stacks of subsequent imaging time points of the same dendritic element. Protrusions from the dendrite that reached a threshold of 0.4 $\mu \mathrm{m}$ were scored as dendritic spines regardless of shape. If spine neck positions differed $>0.5 \mu \mathrm{m}$ in subsequent images, the spine was scored as a new spine. Spines were scored as lost if they fell below the threshold of $0.4 \mu \mathrm{m}$. The survival fraction of spines was calculated as the percentage of remaining spines compared with the first time point: $S F(t)=100$ * $N(t) / N_{0}\left(N_{0}\right.$, spine number at $t_{0} ; N(t)$, surviving spine number at a given time point). The gained and lost fraction $\left(F_{\text {gained }}\right.$ and $\left.F_{\text {lost }}\right)$ of spines was calculated for every time point by dividing the number of gained spines $\left(N_{\text {gained }}\right)$ and the number of lost spines $\left(N_{\text {lost }}\right)$ by the number of present spines $\left(N_{\text {present }} ; F_{\text {gained }}=100 * N_{\text {gained }} / N_{\text {present }} ; F_{\text {lost }}=100 * N_{\text {lost }} / N_{\text {present }}\right)$. Spines that were present for $\leq 4 \mathrm{~d}$ were classified as transient and spines with a lifetime of $\geq 8 \mathrm{~d}$ were classified as persistent. To determine neuronal survival, the overview $z$-stacks of the first and last imaging sessions were compared by manually scrolling through the $z$-stacks. The positions of neurons were compared at the two time points and lost and stable neurons were counted. The presented figures of neurons, dendrites, and spines were median filtered and average-intensity projected.

Quantification and statistics. Quantifications and statistical analysis were performed using GraphPad Prism and Microsoft Excel. All analysis was performed blind to the experimental conditions. One-way and twoway ANOVAs, as well as $t$ tests, were performed to statistically determine significance. All values are displayed as mean \pm SEM if not otherwise stated. 
a

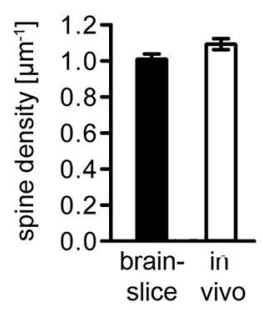

e 10 days post surgery (dps)
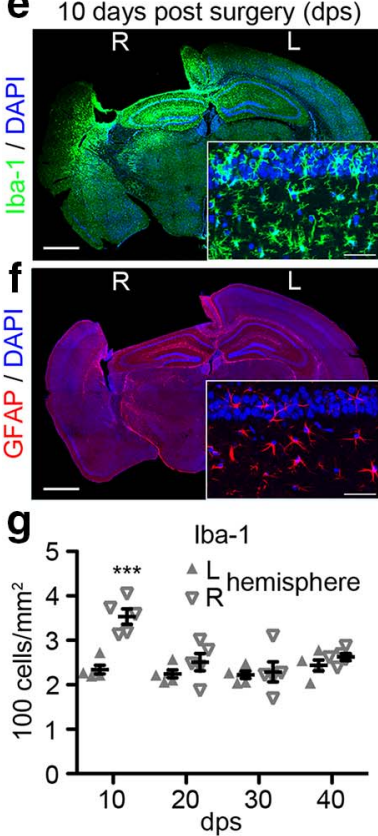

b
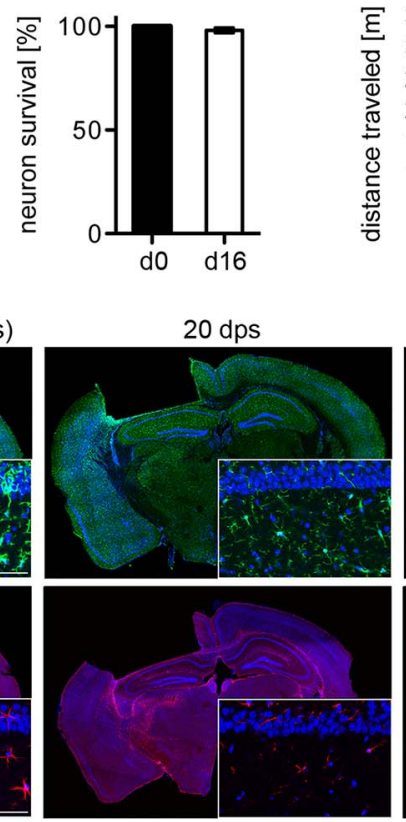

h

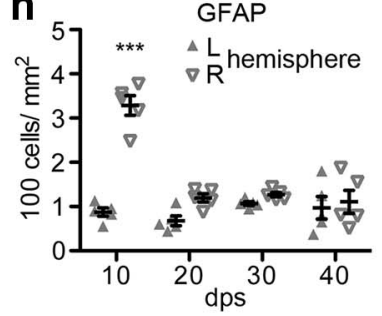

C
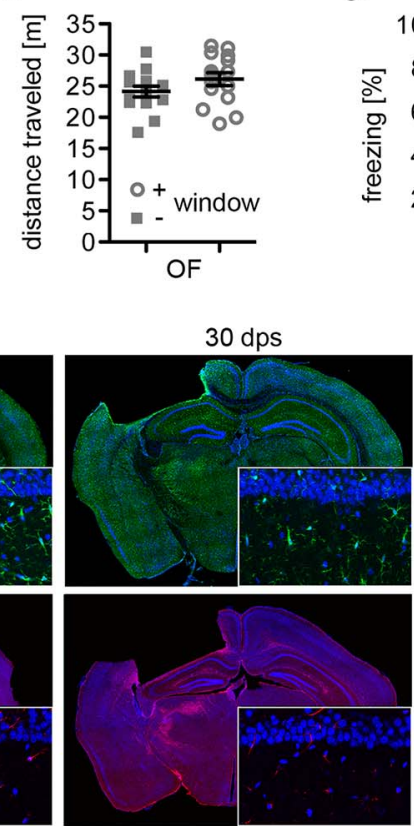

i

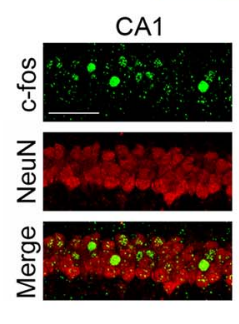

d
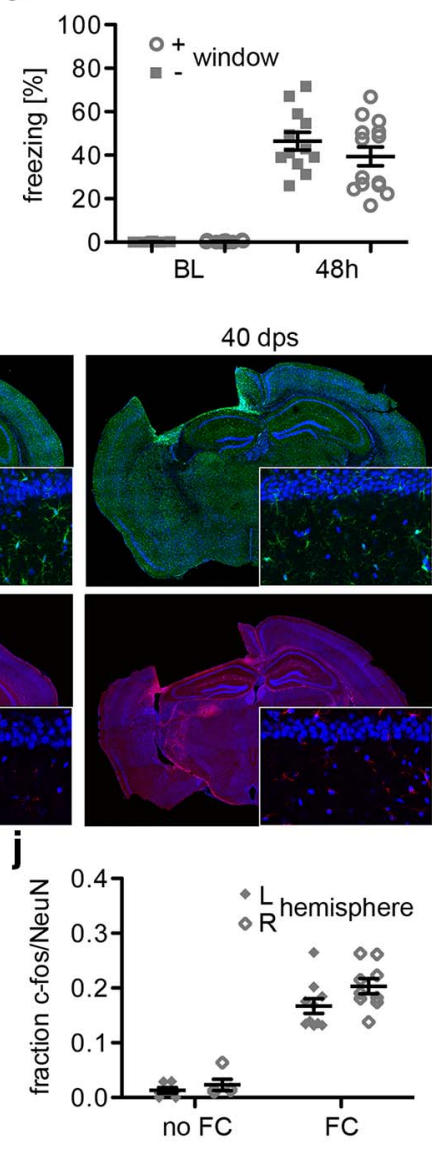

Figure 2. Secondary effects of window implantation. $\boldsymbol{a}$, Spine density of radial oblique dendrites in fixed brain slices without surgery compared with spine densities in vivo ( $p>0.05$, $n=5$ mice/group, $t$ test). $\boldsymbol{b}$, Survival of YFP-positive neurons after $16 \mathrm{~d}$ ( $p>0.05, n=6$ mice, $t$ test). $c$, Open-field (OF) behavior of mice with or without hippocampal window surgery ( $p>0.05, n=14-15$ mice/group, $t$ test). $\boldsymbol{d}$, Freezing rates of mice with or without window surgery at baseline (BL) and $48 \mathrm{~h}$ after fear conditioning $(p>0.05, n=12-14 \mathrm{mice} / \mathrm{group}$, 1-way ANOVA). $\boldsymbol{e}, \boldsymbol{f}$, Microgliosis and astrogliosis at 10, 20, 30, and $40 \mathrm{~d}$ after implanting a hippocampal window. Brain sections were stained with Iba1 (microglia, green), GFAP (astrocytes, red), and DAPI (nuclei, blue). Zoom of hippocampal CA1 layer. $\boldsymbol{g}, \boldsymbol{h}$, Quantification of Iba1-positive ( $\boldsymbol{g}$ ) and GFAP-positive ( $\boldsymbol{h}$ ) cells in the hippocampus of the left (L) and right (R) hemisphere at 10, 20,30, and $40 \mathrm{~d}$ postsurgery (10 d postsurgery: $p<0.001, n=5$ mice/group, 2-way ANOVA, Bonferroni's post-test). $\boldsymbol{i}$, Staining of hippocampal CA1 neurons for c-Fos and NeuN. $j$, Fraction c-Fos-positive of NeuN-positive CA1 neurons in naive mice (no FC) and in mice after fear conditioning (FC), comparing the right (R) hemisphere with implanted window and left (L) hemisphere without window. ( $p>0.05, n=5-10$ mice/group, 2-way ANOVA, Bonferroni's post-tests). Scale bars: $\boldsymbol{e}, \boldsymbol{f}$, overview, $1 \mathrm{~mm} ; \boldsymbol{e}, \boldsymbol{f}, \mathbf{z 0 0 m}, 50 \mu \mathrm{m} ; \boldsymbol{i}$, $50 \mu \mathrm{m}$.

\section{Results}

Spines are structurally plastic in the hippocampus

To examine dendritic spines in the hippocampus over periods of weeks, we adapted the hippocampal window described by Dombeck et al. (2010) and optimized it for high-resolution imaging of subcellular structures, such as dendritic spines (see Materials and Methods). By adjusting the hippocampal window implantation, we were able to perform two-photon in vivo imaging up to a depth of $700 \mu \mathrm{m}$ in the YFP-H mouse line (Fig. 1; Movie 1). This enabled us to image granule cell bodies in the dentate gyrus with cellular resolution (Fig. 1a). Additionally, the resolution was sufficient to visualize dendritic spines on the entire dendritic tree of pyramidal CA1 neurons with basal, radial, and distal dendrites (Fig. $1 a, c$ ). We imaged the very same dendritic elements in stratum radiatum with $4 \mathrm{~d}$ intervals for a period of $16 \mathrm{~d}$ (Fig. $1 b, c)$. The majority of spines remained at their initial positions, while some spines were eliminated and new spines appeared (Fig. 1c,d). A fraction of $96.1 \pm 0.5 \%$ of the spines imaged at the first time point survived $16 \mathrm{~d}$ (Fig. 1e). The average spine density per mouse of radial oblique dendrites ranged between 1.097 and 1.129 $\mu \mathrm{m}^{-1}$ (Fig. 1f) and was stable over the $16 \mathrm{~d}$ observation period since the loss balanced the gain of spines (Fig. $1 f ; p>0.05 ; n=$ 5 mice; two-way ANOVA). The long-term presence of dendritic spines has been shown to correlate with the functional integrity of synapses. In the cortex, dendritic spines with a lifetime of $\leq 4 \mathrm{~d}$ have a low probability of a presynaptic partner, while spines with a lifetime of $\geq 8 \mathrm{~d}$ exhibit a presynapse in all cases (Knott et al., 2006). Thus, categorizing spines into transient and persistent spines can be used as a measure of functional connectivity. We therefore classified the spines into transient (lifetime, $\leq 4 \mathrm{~d}$ ) and persistent (lifetime, $\geq 8 \mathrm{~d}$ ) and followed their fate over time. The density of persistent spines ranged between 1.07 and $1.08 \pm 0.04 \mu \mathrm{m}^{-1}$ and was stable over time (Fig. $1 g$ ). Since there was only a small fraction of newly gained and eliminated spines, the density of transient spines was low, ranging between 0.042 and $0.057 \pm 0.008$ $\mu \mathrm{m}^{-1}$ (Fig. $1 \mathrm{~g}$ ). The real turnover rate of dendritic spines may be higher, since we cannot rule out loss and gain in between the $4 \mathrm{~d}$ intervals that we do not detect. In summary, these results indicate that the majority of spines in radiatum are persistent. However, the network connectivity is plastic 
since new spines are formed and eliminated over a period of weeks.

\section{Secondary effects of hippocampal window implantation}

During the hippocampal window implantation, we removed parts of the cortex, including the somatosensory cortex. To investigate the effects of the surgery on hippocampal spines, we compared the spine density measured with in vivo imaging to the density of radial oblique dendrites in fixed brain slices without surgery. The spine density in fixed brain slices without surgery $\left(1.01 \pm 0.04 \mu \mathrm{m}^{-1}\right)$ was comparable to the spine density obtained with in vivo imaging $\left(1.09 \pm 0.04 \mu \mathrm{m}^{-1}\right.$; Fig. $2 a ; p>0.05$; $n=5$ mice/group; $t$ test). Moreover, a similar spine density of $0.92 \pm 0.15 \mu \mathrm{m}^{-1}$ was measured within a couple of hours after an acute hippocampal window implantation (Mizrahi et al., 2004). This indicates that the spine density is unaffected by the hippocampal window surgery. We further investigated the survival of 1073 YFP-positive CA1 neurons over the $16 \mathrm{~d}$ observation period. We found that the majority $(98.1 \pm 1.1 \%)$ of YFPpositive CA1 neurons survived $16 \mathrm{~d}$ (Fig. $2 b ; p>0.05 ; n=6$ mice; paired $t$ test). Next, we tested whether mice with a hippocampal window exhibited changes in behavior performance. We performed an open-field test to analyze the general mobility capabilities. We did not detect any difference in the distance traveled between mice with or without the hippocampal window (Fig. $2 c$; $p>0.05 ; n=14-15$ mice/group; $t$ test). In the contextual fearconditioning paradigm, mice with or without the hippocampal window showed similarly increased freezing rates (Fig. $2 d ; p>$ $0.05 ; n=12-14$ mice/group; one-way ANOVA with Bonferroni's post-test).

During the first 4 weeks after hippocampal window surgery, we observed insufficient image quality to detect spines. We hypothesized that inflammation might be the reason. Therefore, we analyzed microgliosis and astrogliosis at 10, 20,30, and $40 \mathrm{~d}$ after hippocampal window implantation and compared the cell density in the right and left hippocampus (Fig. $2 e-h$ ). Microgliosis was significantly increased in the right hippocampus below the window $10 \mathrm{~d}$ after surgery and decreased within $20 \mathrm{~d}$, leaving a scar at the surgical border (Fig. 2e,g). Similarly, astrogliosis was significantly increased in the right hippocampus $10 \mathrm{~d}$ postsurgery and decayed after $20 \mathrm{~d}$ (Fig. 2f,h). These data indicate that accumulation of microglia and astrocytes peaked before the improvement of image quality. Hence, we decided to wait for $\geq 4$ weeks after hippocampal window implantation to start imaging.

To test whether both hippocampi were functional after window implantation, we analyzed neuronal activity of CA1 neurons by analyzing the expression of the iEG $c$-fos at $40 \mathrm{~d}$ postsurgery (Fig. 2i). To induce iEG expression, we performed contextual fear conditioning and found significantly increased iEG expression compared with mice without fear conditioning. However, iEG activation was the same in the left and right hemispheres, regardless of window implantation.

\section{Discussion}

We established a hippocampal window two-photon imaging approach to perform long-term high-resolution imaging in the hippocampus. Compared with previous methods (Barretto et al., 2009; Dombeck et al., 2010), we were able to image with subcellular resolution over a period of weeks. Dendritic spines in the neocortex undergo plastic structural changes in vivo (Grutzendler et al., 2002; Trachtenberg et al., 2002; Holtmaat et al., 2005; Keck et al., 2008; Yang et al., 2009; Lai et al., 2012). Here, we demonstrated that spines on radial oblique dendrites of CA1 neu- rons in the hippocampus also exhibit structural plasticity, suggesting a role in experience-dependent circuit rewiring as has been shown in the cortex (Keck et al., 2008). Previous studies in the cortex detected spine densities of $\sim 0.4 \mu \mathrm{m}^{-1}$ on apical dendrites of pyramidal neurons in the somatosensory or visual cortex (Holtmaat et al., 2005; Knott et al., 2006; Keck et al., 2008). In comparison, we found that the spine density in the hippocampus was more than twice as high $\left(\sim 1.1 \mu \mathrm{m}^{-1}\right)$. However, the densities of transient spines in the somatosensory cortex and the hippocampal CA1 region were similar (hippocampus, $0.046 \pm 0.005$ $\mu \mathrm{m}^{-1}$; cortex, $0.063 \pm 0.019 \mu \mathrm{m}^{-1}$; Holtmaat et al., 2005). This result may be a sign of a general dendrite-immanent production and elimination rate of spines that ultimately reflects the plasticity of rewiring on the structural level.

One of the future challenges will be to detect the role of structural plasticity of dendritic spines in hippocampus-dependent behavior, particularly in response to learning and memory processes. Therefore, it is important to determine whether potential side effects of hippocampal window implantation interfere with the function of the hippocampal circuitry. We found comparable spine densities in the hippocampus of mice with and without surgery, indicating that spine densities are unaffected by the window-implantation procedure. However, due to insufficient image quality during the first 4 weeks after hippocampal window implantation, we were unable to analyze spine turnover changes during this period. Therefore, we cannot rule out potential turnover changes that have been controversially discussed during the first 2 weeks after cortical open-skull window implantation (Xu et al., 2007; Holtmaat et al., 2009). The majority of YFP-labeled CA1 neurons $(98.1 \%)$ could be retrieved after a period of $16 \mathrm{~d}$, indicating that the hippocampal neuronal network stays intact. Moreover, we could confirm that window surgery does not interfere with hippocampus-dependent behavior in an open-field and a contextual fear-conditioning paradigm. Indeed, CA1 neurons exhibited comparable iEG expression of $c$-fos in the hippocampus below the window and on the contralateral side. Our experiments also provided important insight into the time course of microgliosis and astrogliosis, which will be useful for future imaging experiments using this approach. While we could not directly attribute the improved optical accessibility to the reduction of astrogliosis and microgliosis, our finding suggests that optimal imaging conditions are encountered after an interval of $\geq 28 \mathrm{~d}$ following surgery. Together, we established long-term in vivo imaging of hippocampal CA1 pyramidal neurons and demonstrated that dendritic spines in the hippocampus can be reliably imaged over weeks. Our finding that dendritic spines show structural plasticity establishes a conceptual and experimental basis for future studies on connectivity changes in response to hippocampus-dependent learning and memory tasks.

\section{References}

Barretto RP, Messerschmidt B, Schnitzer MJ (2009) In vivo fluorescence imaging with high-resolution microlenses. Nat Methods 6:511-512. CrossRef Medline

Bittner T, Fuhrmann M, Burgold S, Ochs SM, Hoffmann N, Mitteregger G, Kretzschmar H, LaFerla FM, Herms J (2010) Multiple events lead to dendritic spine loss in triple transgenic Alzheimer's disease mice. PloS One 5:e15477. CrossRef Medline

Dombeck DA, Harvey CD, Tian L, Looger LL, Tank DW (2010) Functional imaging of hippocampal place cells at cellular resolution during virtual navigation. Nat Neurosci 13:1433-1440. CrossRef Medline

Engert F, Bonhoeffer T (1999) Dendritic spine changes associated with hippocampal long-term synaptic plasticity. Nature 399:66-70. CrossRef Medline

Feng G, Mellor RH, Bernstein M, Keller-Peck C, Nguyen QT, Wallace M, 
Nerbonne JM, Lichtman JW, Sanes JR (2000) Imaging neuronal subsets in transgenic mice expressing multiple spectral variants of GFP. Neuron 28:41-51. CrossRef Medline

Fuhrmann M, Mitteregger G, Kretzschmar H, Herms J (2007) Dendritic pathology in prion disease starts at the synaptic spine. J Neurosci 27: 6224-6233. CrossRef Medline

Gogolla N, Galimberti I, DePaola V, Caroni P (2006) Staining protocol for organotypic hippocampal slice cultures. Nat Protoc 1:2452-2456. CrossRef Medline

Grutzendler J, Kasthuri N, Gan WB (2002) Long-term dendritic spine stability in the adult cortex. Nature 420:812-816. CrossRef Medline

Holtmaat AJ, Trachtenberg JT, Wilbrecht L, Shepherd GM, Zhang X, Knott GW, Svoboda K (2005) Transient and persistent dendritic spines in the neocortex in vivo. Neuron 45:279-291. CrossRef Medline

Holtmaat A, Bonhoeffer T, Chow DK, Chuckowree J, De Paola V, Hofer SB, Hübener M, Keck T, Knott G, Lee WC, Mostany R, Mrsic-Flogel TD, Nedivi E, Portera-Cailliau C, Svoboda K, Trachtenberg JT, Wilbrecht L (2009) Long-term, high-resolution imaging in the mouse neocortex through a chronic cranial window. Nat Protoc 4:1128-1144. CrossRef Medline

Hübener M, Bonhoeffer T (2010) Searching for engrams. Neuron 67:363371. CrossRef Medline

Keck T, Mrsic-Flogel TD, Vaz Afonso M, Eysel UT, Bonhoeffer T, Hübener M (2008) Massive restructuring of neuronal circuits during functional reorganization of adult visual cortex. Nat Neurosci 11:1162-1167. CrossRef Medline

Knott GW, Holtmaat A, Wilbrecht L, Welker E, Svoboda K (2006) Spine growth precedes synapse formation in the adult neocortex in vivo. Nat Neurosci 9:1117-1124. CrossRef Medline

Lai CS, Franke TF, Gan WB (2012) Opposite effects of fear conditioning and extinction on dendritic spine remodelling. Nature 483:87-91. CrossRef Medline

Mizrahi A, Crowley JC, Shtoyerman E, Katz LC (2004) High-resolution in vivo imaging of hippocampal dendrites and spines. J Neurosci 24:31473151. CrossRef Medline

Nägerl UV, Eberhorn N, Cambridge SB, Bonhoeffer T (2004) Bidirectional activity-dependent morphological plasticity in hippocampal neurons. Neuron 44:759-767. CrossRef Medline

Spires TL, Meyer-Luehmann M, Stern EA, McLean PJ, Skoch J, Nguyen PT, Bacskai BJ, Hyman BT (2005) Dendritic spine abnormalities in amyloid precursor protein transgenic mice demonstrated by gene transfer and intravital multiphoton microscopy. J Neurosci 25:7278-7287. CrossRef Medline

Trachtenberg JT, Chen BE, Knott GW, Feng G, Sanes JR, Welker E, Svoboda $\mathrm{K}$ (2002) Long-term in vivo imaging of experience-dependent synaptic plasticity in adult cortex. Nature 420:788-794. CrossRef Medline

Tsai J, Grutzendler J, Duff K, Gan WB (2004) Fibrillar amyloid deposition leads to local synaptic abnormalities and breakage of neuronal branches. Nat Neurosci 7:1181-1183. CrossRef Medline

Wiegert JS, Oertner TG (2013) Long-term depression triggers the selective elimination of weakly integrated synapses. Proc Natl Acad Sci U S A 110: E4510-E4519. CrossRef Medline

Xu HT, Pan F, Yang G, Gan WB (2007) Choice of cranial window type for in vivo imaging affects dendritic spine turnover in the cortex. Nat Neurosci 10:549-551. CrossRef Medline

Yang G, Pan F, Gan WB (2009) Stably maintained dendritic spines are associated with lifelong memories. Nature 462:920-924. CrossRef Medline

Zhou Q, Homma KJ, Poo MM (2004) Shrinkage of dendritic spines associated with long-term depression of hippocampal synapses. Neuron 44: 749-757. CrossRef Medline 